\title{
Threshold Based Relay Selection in Cooperative Wireless Networks
}

\author{
Furuzan Atay Onat ${ }^{1}$, Yijia Fan ${ }^{2}$, Halim Yanikomeroglu ${ }^{1}$, and H. Vincent Poor $^{2}$ \\ ${ }^{1}$ Broadband Communications and Wireless Systems (BCWS) Centre, \\ Department of Systems and Computer Engineering Carleton University, Ottawa, Canada \\ E-mail: e-mail: \{furuzan, halim\} esce.carleton.ca \\ ${ }^{2}$ Department of Electrical Engineering, Princeton University, Princeton, NJ, USA \\ e-mail: $\{$ yijiafan, poor $\}$ @princeton.edu
}

\begin{abstract}
This paper considers two-hop cooperative relaying using multiple relays and proposes a threshold based relay selection protocol. In this protocol the relays are selected among those having received SNR higher than a threshold value. The relay selection is performed by the destination based on the received SNRs at the destination during the last hop. The exact bit error rate of this protocol is derived and it is shown that it achieves full diversity order. Unlike some other full diversity achieving protocols in the literature, the requirement that the exact/average SNRs of the source-relay links be known at the destination is eliminated using an appropriate SNR threshold.
\end{abstract}

\section{INTRODUCTION}

Cooperative relaying can induce spatial diversity in wireless networks without any reliance on multiple antennas. Various decode-and-forward protocols have been proposed based on selective relaying, distributed space-time coding and relay selection, and have been shown to achieve full diversity [1]-[3]. Recently, detection aspects of cooperative relaying have been analyzed [4]-[8]. These works study digital (or demodulate-and-forward) cooperative relaying protocols, in which the relaying does not rely on any error correction or detection codes. Such protocols are particularly attractive for wireless sensor networks, for which coded transmission can be costly due to severe energy limitations.

Unlike ideal decode-and-forward relaying, in digital relaying the relays can forward erroneous information, and with a conventional combining scheme such as Maximal Ratio Combining (MRC), these errors propagate to the destination, causing end-to-end (e2e) detection errors. Existing techniques for mitigating error propagation can be classified into two groups. The first of these comprises selective and adaptive relaying techniques, which include link adaptive relaying (LAR) [4], [5] and threshold digital relaying (TDR) [9]-[11]. Both techniques use link SNRs to evaluate the reliability of the data received by the relay. In TDR a relay forwards the received data only when its received SNR is above a threshold value. In LAR the relay transmits with a fraction $\alpha$ of its maximum transmit power, where $\alpha$ depends on the source-relay and relay-destination SNRs. In [4], a function for calculating $\alpha$

This research was supported by the U.S. National Science Foundation under Grants ANI-03-38807 and CNS-06-25637. is provided and the resulting scheme is shown to achieve full diversity if the relays are capable of adjusting their transmit powers continuously. However, the proposed function cannot provide diversity if reduced to two power levels, i.e., on/off power adaptation. TDR can also be viewed as on/off power adaptation, and it is shown in [11] that it can achieve full diversity in the single relay case.

The second approach to mitigate error propagation is to develop better combining schemes for the destination. These schemes take the possibility of error propagation into account and require the relays to send their source-relay link SNRs (average or instantaneous) to the destination.

In [7], Wang et al. assume that the destination knows the exact source-relay SNR, and derive a linear combiner, called Cooperative MRC (C-MRC), that approximates the Maximum Likelihood (ML) receiver. This receiver achieves full diversity at the expense of increased signaling to convey the first hop SNR information to the destination. In [6], the authors propose a piece-wise linear receiver approximating the ML detector that requires knowledge of the average SNRs of the first hop. Conveying the average link SNRs is less costly than conveying the exact SNR. However, this protocol cannot achieve full diversity for more than one relay. The protocol we consider requires minimal information on the first hop and still achieves full diversity.

In this paper, we propose the Threshold based Relay Selection Cooperation (TRSC) protocol, which generalizes threshold digital relaying to multiple relays. In this protocol only the relays whose received SNRs are larger than a threshold, which we call reliable relays, are allowed to retransmit. Consider a multiple parallel relay configuration as shown in Fig. 1. With $M$ parallel relays there is a potential to achieve $M+1$ orders of diversity by combining signals from the source and the relays. Our protocol employs selection combining at the destination based only on the relay-destination and source-destination link SNRs. In bandwidth limited scenarios, with channel estimation of these links and feedback from the destination, selection can be done prior to the relay transmissions as performed in [3], [12]. Then, only the selected relay, the one with the largest SNR to the destination, retransmits, thereby reducing the bandwidth expansion. In the absence of such feedback, all the reliable relays can retransmit sequentially and the same bit 
error rate (BER) performance is achieved. A related protocol has been proposed in [8]. In this protocol, the relay selection is performed based on the equivalent e2e BER of each relay channel. This protocol can be viewed as a selection version of C-MRC of [7]. As in C-MRC, it requires the destination to obtain the channel coefficients of the first and second hops, or their product in the case of a simpler scheme, to make relay selection. However, in TRSC, the information passed from the relay to the destination regarding the first hop is limited to whether the relay is reliable or not, which can be represented by a single bit.

The rest of this paper is organized as follows. In Section II, we describe the system model and the TRSC protocol. In Section III, we derive the e2e BER of the protocol, and in Section IV we show that the protocol achieves full diversity using a threshold function we propose. We present some numerical results in Section V and conclude in Section VI with a summary of our results.

\section{System ModeL}

A network as shown in Fig. 1 is considered in which a source node $S$ communicates with a destination node $D$ with the assistance of $M$ relays denoted by $R_{1}, R_{2}, \ldots R_{M}$. All links experience independent Rayleigh fading. We assume a general modulation scheme for which the bit error probability can be expressed as $P_{b}(\gamma) \approx b \operatorname{erfc}(\sqrt{a \gamma})$, where $a, b>0$. Note that typically $b$ depends on the minimum distance in the constellation and $a$ depends on the number of neighbors with minimum distance; the bit error probability of most practical modulation schemes can be approximated by selecting $(b, a)$. For instance, for BPSK, $(b, a)=(0.5,1)$ gives the exact BER, and for M-PSK, $(b, a)=\left(1 / \log _{2}(M), \log _{2}(M) \sin ^{2}(\pi / M)\right)$ can be used to approximate the BER. Based on this general $P_{b}$ expression, the average bit error probability under Rayleigh fading is calculated as [13, pg. 185]

$$
\bar{P}_{b}(\bar{\gamma})=\mathbb{E}_{\gamma}[b \operatorname{erfc}(\sqrt{a \gamma})]=b\left[1-\sqrt{\frac{a \bar{\gamma}}{1+a \bar{\gamma}}}\right] .
$$

Some of our derivations are even more general; they are given in terms of $P_{b}$ and $\bar{P}_{b}$, and can be evaluated for any modulation scheme.

The SNRs of the $S-D, S-R_{i}$ and $R_{i}-D$ links are denoted by $\gamma_{s d}, \gamma_{s r, i}$, and $\gamma_{r d, i}$, respectively. To simplify the analysis, we assume that all the relays have the same average SNRs to the source and to the destination, i.e., $\bar{\gamma}_{s r, i}=\bar{\gamma}_{s r}$ and $\bar{\gamma}_{r d, i}=\bar{\gamma}_{r d}$ for $i=1,2, \ldots, M$. Hence, the link SNRs are characterized by $\bar{\gamma}_{s d}, \bar{\gamma}_{s r}$, and $\bar{\gamma}_{r d}$.

We consider a two-phase protocol. In the first phase the source transmits while all the relays and the destination listen. Then each relay $R_{i}$ decides independently whether its detection is reliable by comparing its received SNR $\gamma_{s r, i}$ to a threshold value. Those relays whose received SNRs are larger than the threshold are called reliable relays. Each reliable relay informs the destination by sending a short message. Let $N_{r}$ denote the number of reliable relays. The destination, then, makes a decision based on the SNRs of the reliable relays and the source to the destination ${ }^{1}$, i.e., $\gamma_{s d}$ and $\gamma_{r d, 1}, \ldots, \gamma_{r d, N_{r}}$.

\footnotetext{
${ }^{1}$ We re-index the reliable relays to simplify the notation.
}

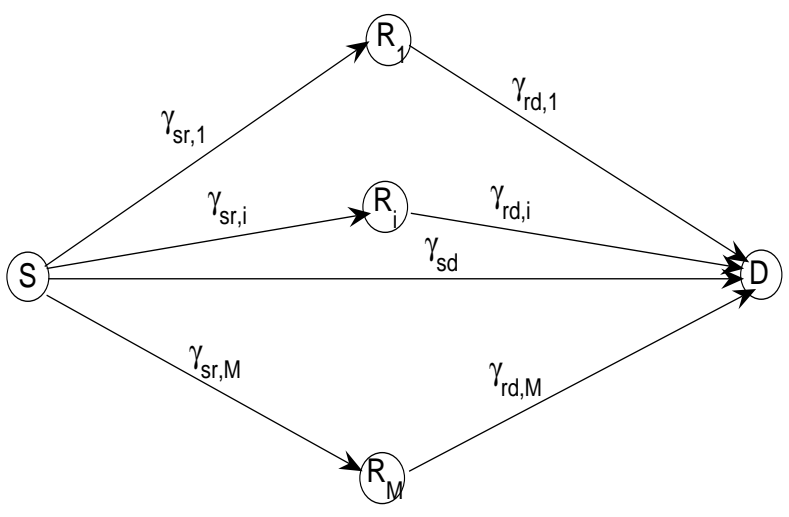

Fig. 1. The network model.

Among $N_{r}+1$ branches $D$ selects the one with the largest SNR. If the branch from the source is selected, the relays do not transmit and the source transmits the next data. Otherwise, the selected reliable relay transmits and $D$ performs detection based on the selected branch only. As noted above, we call this protocol the Threshold based Relay Selection Cooperation (TRSC) protocol.

In this protocol the information passed from the relay to the destination regarding the first hop is limited to whether the relay is a reliable relay or not, which can be represented by a single bit. We assume that each reliable relay sends a short packet while the other relays remain silent. The destination can also estimate the values of $\gamma_{r d, i}$ for all the reliable relays from these transmissions.

For comparison we study the performance of schemes in which the destination also has either the instantaneous $S-R_{i}$ SNRs $\gamma_{s r, i}$ for all links or their average $\bar{\gamma}_{s r}$.

We use the following definitions and notation in the rest of the paper.

Definition 1: Let $f$ and $g$ be two positive functions defined on the real numbers. We say $f=O(g)$, if $\lim \sup _{x \rightarrow \infty} \frac{f(x)}{g(x)}<$ $\infty$.

Definition 2: Two functions $f$ and $g$ are called asymptotically equivalent, written $f \sim g$, if $\lim _{x \rightarrow \infty} \frac{f(x)}{g(x)}=1$.

We adopt the following definition of diversity order given in $[14]^{2}: d=-\lim _{\mathrm{SNR} \rightarrow \infty}(\log (\mathrm{BER}) / \log (\mathrm{SNR}))$.

\section{END-TO-END (E2E) BER OF THE TRSC}

In this section, we derive the e2e BER of the system described in Section II. Since all the relays are assumed to be identical in their average SNR to the relay and the destination, the optimal value of their thresholds must be the same. Hence, we derive the e2e BER of the system for a given common threshold $\gamma_{t}$ for all relays. Then the e2e BER is given by

$$
\mathrm{BER}_{e 2 e}=\sum_{i=0}^{M} \mathbb{P}\left(N_{r}=i\right) \mathbb{P}\left(\mathcal{E}_{e 2 e} \mid N_{r}=i\right)
$$

\footnotetext{
${ }^{2}$ Throughout this paper all the logarithms are in the natural base unless indicated otherwise.
} 
where

$$
\mathbb{P}\left\{N_{r}=i\right\}=\left(\begin{array}{c}
M \\
i
\end{array}\right)\left(e^{-\gamma_{t} / \bar{\gamma}_{s r}}\right)^{i}\left(1-e^{-\gamma_{t} / \bar{\gamma}_{s r}}\right)^{M-i} .
$$

For $N_{r}=0$, the destination detects based on the direct link only and, thus, $\mathbb{P}\left(\mathcal{E}_{e 2 e} \mid N_{r}=0\right)=\bar{P}_{b}\left(\bar{\gamma}_{s d}\right)$. For $N_{r} \geq 1$, let $\mathcal{A}_{s}$ denote the event that the destination selects the signal received from the source and $\mathcal{A}_{r, k}$ denote the event that the destination selects the signal from the $k$-th reliable relay $(k \in$ $\left.\left\{1, \ldots, N_{r}\right\}\right)$, respectively:

$$
\begin{aligned}
\mathcal{A}_{s} & =\left\{\gamma_{s d}>\gamma_{r d, j}, \forall j \in\left\{1, \ldots, N_{r}\right\}\right\}, \text { and } \\
\mathcal{A}_{r, k} & =\left\{\gamma_{r d, k}>\gamma_{s d}, \gamma_{r d, k}>\gamma_{r d, j}, \forall j \in\left\{1, \ldots, N_{r}\right\}, j \neq k\right\} .
\end{aligned}
$$

Then, the e2e BER conditioned on the number of reliable relays is equal to

$$
\begin{aligned}
\mathbb{P}\left(\mathcal{E}_{e 2 e} \mid N_{r}=i\right)=\mathbb{P}\left\{\mathcal{E}_{e 2 e} \mid \mathcal{A}_{s}, N_{r}=i\right\} \mathbb{P}\left\{\mathcal{A}_{s} \mid N_{r}=i\right\} \\
+\sum_{k=1}^{i} \mathbb{P}\left\{\mathcal{E}_{e 2 e} \mid \mathcal{A}_{r, k}, N_{r}=i\right\} \mathbb{P}\left\{\mathcal{A}_{r, k} \mid N_{r}=i\right\} .
\end{aligned}
$$

Since all relays are assumed to be identical in their average SNRs to the source and the destination, the terms included in $\mathcal{A}_{r, k}$ are the same for all $k$ and the index $k$ can be dropped. When the destination selects the source signal, its bit error rate depends only on the source-destination link. However, if the destination selects reliable relay $j$, it will have a bit error if either the $S-R_{j}$ link or the $R_{j}-D$ link has a bit error:

$$
\mathbb{P}\left\{\mathcal{E}_{e 2 e} \mid \mathcal{A}_{s}, N_{r}=i\right\}=\mathbb{P}\left\{\mathcal{E}_{s d} \mid \mathcal{A}_{s}, N_{r}=i\right\}
$$

and

$$
\begin{aligned}
\mathbb{P}\left\{\mathcal{E}_{e 2 e} \mid \mathcal{A}_{r}, N_{r}=i\right\} \\
\quad=\mathbb{P}\left\{\mathcal{E}_{r d} \mid \mathcal{A}_{r}, N_{r}=i\right\}\left(1-\mathbb{P}\left\{\mathcal{E}_{s r} \mid \gamma_{s r}>\gamma_{t}\right\}\right) \\
\quad+\left(1-\mathbb{P}\left\{\mathcal{E}_{r d} \mid \mathcal{A}_{r}, N_{r}=i\right\}\right) \mathbb{P}\left\{\mathcal{E}_{s r} \mid \gamma_{s r}>\gamma_{t}\right\} .
\end{aligned}
$$

The probability of bit error at a reliable relay is given by [9]

$$
\begin{aligned}
\mathbb{P}\left\{\mathcal{E}_{s r} \mid \gamma_{s r}>\gamma_{t}\right\} & =b\left[\operatorname{erfc}\left(\sqrt{a \gamma_{t}}\right)-e^{\gamma_{t} / \bar{\gamma}_{s r}} \sqrt{\frac{a \bar{\gamma}_{s r}}{1+a \bar{\gamma}_{s r}}}\right. \\
& \left.\times \operatorname{erfc}\left(\sqrt{\gamma_{t}\left(a+\frac{1}{\bar{\gamma}_{s r}}\right)}\right)\right] .
\end{aligned}
$$

Substituting (4) and (5) into (3), we obtain the e2e BER conditioned on $N_{r}$ as

$$
\begin{aligned}
\mathbb{P}\left(\mathcal{E}_{e 2 e} \mid N_{r}=i\right) & =\mathbb{P}\left\{\mathcal{E}_{s d}, \mathcal{A}_{s} \mid N_{r}=i\right\}+i\left(\mathbb{P}\left\{\mathcal{E}_{r d}, \mathcal{A}_{r} \mid N_{r}=i\right\}\right. \\
& +\mathbb{P}\left\{\mathcal{E}_{r d}, \mathcal{A}_{r} \mid N_{r}=i\right\}\left(1-2 \mathbb{P}\left\{\mathcal{E}_{s r} \mid \gamma_{s r}>\gamma_{t}\right\}\right) \\
& \left.+\mathbb{P}\left\{\mathcal{A}_{r} \mid N_{r}=i\right\} \mathbb{P}\left\{\mathcal{E}_{s r} \mid \gamma_{s r}>\gamma_{t}\right\}\right) .
\end{aligned}
$$

The probability that a particular reliable relay is selected by the destination is equal to

$$
\mathbb{P}\left\{\mathcal{A}_{r} \mid N_{r}=i\right\}=\frac{1}{i}\left(1-\sum_{j=0}^{i}\left(\begin{array}{l}
i \\
j
\end{array}\right)(-1)^{j} \frac{1}{1+j\left(\bar{\gamma}_{s d} / \bar{\gamma}_{r d}\right)}\right) .
$$

The terms $\mathbb{P}\left\{\mathcal{E}_{s d}, \mathcal{A}_{s} \mid N_{r}=i\right\}$ and $\mathbb{P}\left\{\mathcal{E}_{r d}, \mathcal{A}_{r} \mid N_{r}=i\right\}$ are given by

$$
\begin{aligned}
\mathbb{P}\left\{\mathcal{E}_{s d}, \mathcal{A}_{s} \mid N_{r}=i\right\}=\sum_{j=0}^{i}\left\{\left(\begin{array}{l}
i \\
j
\end{array}\right)(-1)^{j} \frac{\bar{\gamma}_{r d}}{j \bar{\gamma}_{s d}+\bar{\gamma}_{r d}}\right. \\
\left.\times \bar{P}_{b}\left(\frac{\bar{\gamma}_{s d} \bar{\gamma}_{r d}}{j \bar{\gamma}_{s d}+\bar{\gamma}_{r d}}\right)\right\},
\end{aligned}
$$

and

$$
\begin{gathered}
\mathbb{P}\left\{\mathcal{E}_{r d}, \mathcal{A}_{r} \mid N_{r}=i\right\}=\sum_{j=0}^{i-1}\left\{( \begin{array} { c } 
{ i - 1 } \\
{ j }
\end{array} ) ( - 1 ) ^ { j } \left[\frac{1}{j+1} \bar{P}_{b}\left(\frac{\bar{\gamma}_{r d}}{j+1}\right)\right.\right. \\
\left.\left.-\frac{\bar{\gamma}_{s d}}{\bar{\gamma}_{s d}(j+1)+\bar{\gamma}_{r d}} \bar{P}_{b}\left(\frac{\bar{\gamma}_{s d} \bar{\gamma}_{r d}}{\bar{\gamma}_{s d}(j+1)+\bar{\gamma}_{r d}}\right)\right]\right\} .
\end{gathered}
$$

Derivation of (8)-(10) is straightforward from the properties of ordered random variables and, hence, is not included here. By substituting (8)-(10) into (7), and then substituting (3), (7) into (2), we obtain an exact expression for the e2e BER of the threshold based relay selection protocol described in Section II.

\section{DIVERSITY ORDER OF TRSC}

In this section, we consider a modulation scheme with $P_{b}(\gamma)=b \operatorname{erfc}(\sqrt{a \gamma})$. Based on the insight from the e2e BER minimizing threshold derived in [11], for a network with $M$ relays we propose to use a threshold function in the form of $\log \left(c_{1} \mathrm{SNR}^{M / a}\right)$, where $c_{1}$ is a positive constant. Next, we show that TRSC can achieve full diversity with the proposed threshold function.

The e2e BER is given in (2). For the first term we have

$$
\mathbb{P}\left\{N_{r}=i\right\}=\left(\begin{array}{c}
M \\
i
\end{array}\right)\left(e^{-\gamma_{t} / \bar{\gamma}_{s r}}\right)^{i}\left(1-e^{-\gamma_{t} / \bar{\gamma}_{s r}}\right)^{M-i} .
$$

With the proposed threshold, as SNR $\rightarrow \infty$ we have

$$
\begin{aligned}
e^{-\gamma_{t} / \bar{\gamma}_{s r}} & =e^{-\log \left(c_{1} \mathrm{SNR}^{M / a}\right) /\left(\lambda_{s r} \mathrm{SNR}\right)} \sim 1, \\
\left(1-e^{-\gamma_{t} / \bar{\gamma}_{s r}}\right) & =1-e^{-\log \left(c_{1} \mathrm{SNR}^{M / a}\right) /\left(\lambda_{s r} \mathrm{SNR}\right)} \\
& \sim \frac{\log \left(c_{1} \mathrm{SNR}^{M / a}\right)}{\lambda_{s r} \mathrm{SNR}} .
\end{aligned}
$$

Thus, $\mathbb{P}\left\{N_{r}=i\right\}$ is of order

$$
\mathbb{P}\left\{N_{r}=i\right\}=O\left(\log \left(\mathrm{SNR}^{M / a}\right)^{M-i} / \mathrm{SNR}^{M-i}\right) .
$$

Next, we study how fast the term $\mathbb{P}\left\{\mathcal{E}_{e 2 e} \mid N_{r}=i\right\}$ (given in (7)) decays with increasing SNR.

Lemma 1 (Asymptotic behavior of $\mathbb{P}\left\{\mathcal{E}_{e 2 e} \mid N_{r}=i\right\}$ ): With the proposed threshold $\gamma_{t}^{*}=\log \left(c_{1} \mathrm{SNR}^{M / a}\right)$, we have $\mathbb{P}\left\{\mathcal{E}_{e 2 e} \mid N_{r}=i\right\}=O\left(1 / \mathrm{SNR}^{i+1}\right)$.

See Appendix I for the proof.

Combining the result of Lemma 1 with (11), we observe that in (2) the term with index $i$, i.e., $\mathbb{P}\left\{N_{r}=i\right\} \mathbb{P}\left\{\mathcal{E}_{e 2 e} \mid N_{r}=i\right\}$ decreases as $O\left(\log \left(\mathrm{SNR}^{M / a}\right)^{M-i} / \mathrm{SNR}^{M+1}\right)$. The order of the sum of these $M+1$ terms is determined by the term that has the slowest decay, which is the term with index $i=0$. Hence,

$$
\mathbb{P}\left\{\mathcal{E}_{e 2 e}\right\}=O\left(\log \left(\mathrm{SNR}^{M / a}\right)^{M} / \mathrm{SNR}^{M+1}\right) .
$$


We observe that while the $M+1$ order diversity achieved by conventional diversity combining schemes will decrease as $1 / \mathrm{SNR}^{M+1}$, the cooperative diversity achieved by the TRSC protocol has a decay of $O\left(\log \left(\mathrm{SNR}^{M / a}\right)^{M} / \mathrm{SNR}^{M+1}\right)$. However, at large SNR the log term becomes insignificant and the diversity order is equal to

$$
d=-\lim _{\mathrm{SNR} \rightarrow \infty} \frac{\log \left(\mathbb{P}\left\{\mathcal{E}_{e 2 e}\right\}\right)}{\log (\mathrm{SNR})}=M+1 .
$$

\section{Numerical Performance Results}

In this section we compare the e2e BER of TRSC to two other Relay Selection Cooperation (RSC) protocols that are described below. The first one is $R S C$-inst and is very similar to the $\mathrm{C}-\mathrm{MRC}$ with relay selection introduced in $[8]^{3}$. In RSCinst the relay is selected based on the equivalent BER of branches. The equivalent BER of relay $k$ is given by

$$
\begin{aligned}
P_{k}^{i n s t}\left(\gamma_{s r, k}, \gamma_{r d, k}\right) & =P_{b}\left(\gamma_{s r, k}\right)\left(1-P_{b}\left(\gamma_{r d, k}\right)\right) \\
& +P_{b}\left(\gamma_{r d, k}\right)\left(1-P_{b}\left(\gamma_{s r, k}\right)\right), k=1, \ldots, M,
\end{aligned}
$$

and the destination selects the branch with the minimum equivalent BER. The second protocol we compare to is TSC$a v r$ in which the destination has no knowledge of $\gamma_{s r, k}$ values and the relay selection is based on $\bar{\gamma}_{s r, k}, \gamma_{s d}$ and $\gamma_{r d, k}$ values. Then, the equivalent BER of relay $k$ is given by

$$
\begin{aligned}
P_{k}^{a v r}\left(\bar{\gamma}_{s r, k}, \gamma_{r d, k}\right) & =\bar{P}_{b}\left(\bar{\gamma}_{s r, k}\right)\left(1-P_{b}\left(\gamma_{r d, k}\right)\right) \\
& +P_{b}\left(\gamma_{r d, k}\right)\left(1-\bar{P}_{b}\left(\bar{\gamma}_{s r, k}\right)\right), k=1, \ldots, M .
\end{aligned}
$$

Note that for both RSC-inst and RSC-avg, $P_{0}=P_{b}\left(\gamma_{s d}\right)$.

While RSC-inst is the selection version of the C-MRC of [7], RSC-avg can be viewed as the selection version of the PL receiver of [6].

For numerical results, we consider a symmetric network scenario, where all average link SNRs are the same and are equal to $\bar{\gamma}$. The e2e BER of TRSC is computed from the analytical expression of Section III and the threshold values are determined from the numerical minimization of this expression. Fig. 2 shows the e2e BER of different protocols as a function of $\bar{\gamma}$ for $M=1$ relay. In this figure, TRSC and RSC-avr perform similarly, while RSC-inst performs slightly better than these two protocols. For $M=1$, all protocols achieve full diversity gain as observed from the slopes of the BER curves. However, as we increase the number of relays to $M=2$, RSC-avr cannot deliver full diversity. In fact, by analyzing RSC-avr for different $M$ values, we observe that the diversity order of RSC-avr is limited to 2 . The TRSC achieves full diversity, in accordance with our claims in Section IV, without instantaneous $S-R$ SNR knowledge at the destination. We conclude that TRSC constitutes a good tradeoff between performance and signaling overhead since it performs close to RSC-inst with less complexity. In Fig. 4, we show the threshold values used by TRSC to minimize e2e BER. It is seen that the optimal threshold increases with increasing number of relays. As a function of average

\footnotetext{
${ }^{3}$ The only difference is that the scheme in [8] combines the direct signal with one of the relay signals, whereas we select either one of the relays or the destination.
}

SNR, the optimal threshold increases logarithmically. Finding optimal thresholds analytically remains a challenging problem for future work.

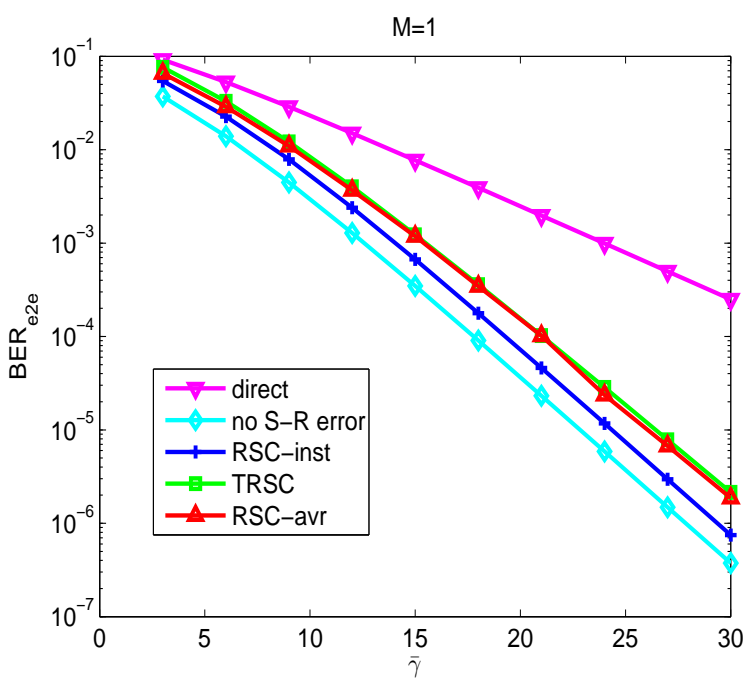

Fig. 2. The e2e BER for all relaying protocols for $M=1$ relay. The BER of direct transmission and the BER in the absence of errors in the $S-R_{i}$ links are also shown as reference curves.

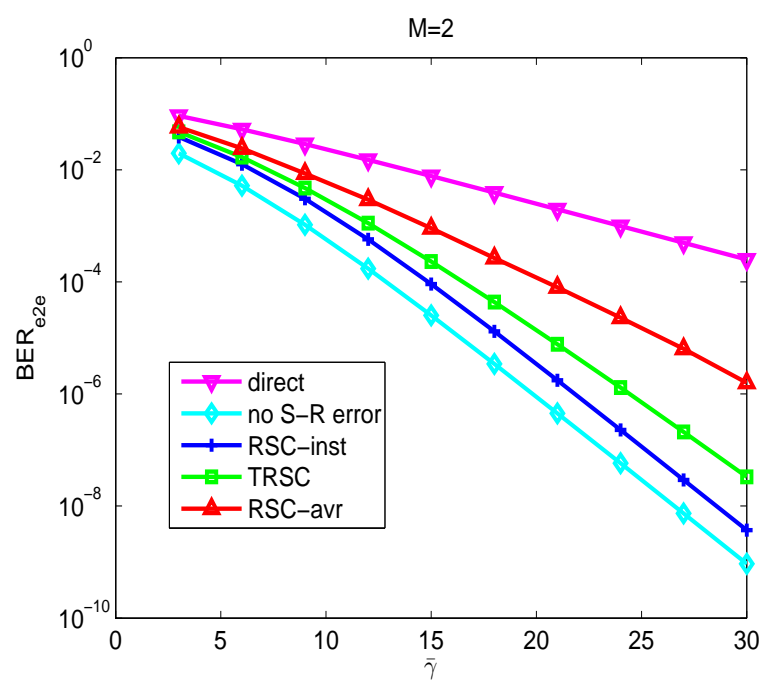

Fig. 3. The e2e BER for all relaying protocols for $M=2$ relays. The BER of direct transmission and the BER in the absence of errors in the $S-R_{i}$ links are also shown as reference curves.

\section{CONCLUSIONS}

In this paper, we have proposed a threshold based relay selection protocol for two hop, multi-relay cooperative communication. This protocol requires minimal information about the SNRs of the source-relay links. We have proposed a threshold function that increases logarithmically with the link SNRs and linearly with the number of relays and we have shown that, with a threshold of this form, our protocol achieves full diversity. We have also presented performance results 


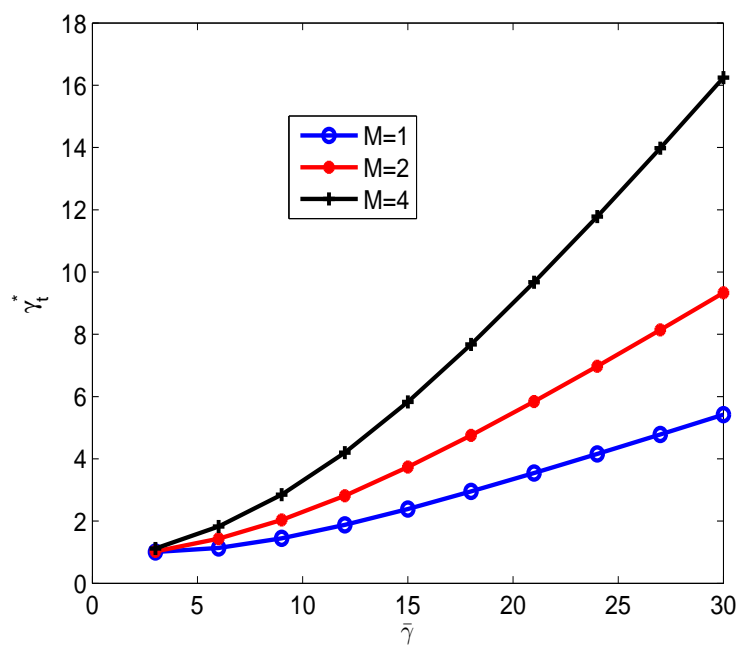

Fig. 4. Threshold values that minimize e2e BER of TRSC in symmetric networks with different numbers $M$ of relays.

in which the thresholds are determined through numerical optimization and have compared the BER of our protocol to similar protocols found in the literature.

\section{APPENDIX I}

\section{PROOF OF LEMMA 1}

We prove this lemma by analyzing the orders of terms in (7) as SNR $\rightarrow \infty$.

Part 1: Let us first analyze the asymptotic behavior of $\mathbb{P}\left\{\mathcal{E}_{s d}, \mathcal{A}_{s} \mid N_{r}=i\right\}$ and $\mathbb{P}\left\{\mathcal{E}_{r d}, \mathcal{A}_{r} \mid N_{r}=i\right\}$. In the absence of errors at the reliable relays the bit error probability at the destination would be equal to the performance of $(i+1)$ branch selection combining (SC), where one of the branches has average SNR of $\bar{\gamma}_{s d}$, and the rest have $\bar{\gamma}_{r d}$. The probability of bit error of SC can be expressed as

$$
\bar{P}_{b}^{S C}\left(i, \bar{\gamma}_{s d}, \bar{\gamma}_{r d}\right)=\mathbb{P}\left\{\mathcal{E}_{s d}, A_{s} \mid N_{r}=i\right\}+i \mathbb{P}\left\{\mathcal{E}_{r d}, A_{r} \mid N_{r}=i\right\} .
$$

Hence, $\mathbb{P}\left\{\mathcal{E}_{s d}, A_{s} \mid N_{r}=i\right\} \leq \bar{P}_{b}^{S C}\left(i, \bar{\gamma}_{s d}, \bar{\gamma}_{r d}\right)$ and $\mathbb{P}\left\{\mathcal{E}_{r d}, A_{r} \mid N_{r}=i\right\} \leq \bar{P}_{b}^{S C}\left(i, \bar{\gamma}_{s d}, \bar{\gamma}_{r d}\right)$. Since $\mathrm{SC}$ is known to achieve diversity order equal to the number of its branches, we conclude that both $\mathbb{P}\left\{\mathcal{E}_{s d}, \mathcal{A}_{s} \mid N_{r}=i\right\}$ and $\mathbb{P}\left\{\mathcal{E}_{r d}, \mathcal{A}_{r} \mid N_{r}=i\right\}$ decrease at least as fast as $1 / \mathrm{SNR}^{i+1}$ : $\mathbb{P}\left\{\mathcal{E}_{s d}, A_{s} \mid N_{r}=i\right\}=O\left(1 / \mathrm{SNR}^{i+1}\right)$ and $\mathbb{P}\left\{\mathcal{E}_{r d}, A_{r} \mid N_{r}=\right.$ $i\}=O\left(1 / \mathrm{SNR}^{i+1}\right)$.

Part 2: Now, let us examine the order of the term $\mathbb{P}\left\{\mathcal{E}_{s r} \mid \gamma_{s r}>\right.$ $\left.\gamma_{t}\right\}$ if $\gamma_{t}=\log \left(c_{1} \mathrm{SNR}^{M / b}\right)$. The analysis closely follows that given in [11] for $M=1$ relay. In [11] for BPSK and any threshold $\gamma_{t}$ it is shown that $\mathbb{P}\left\{\mathcal{E}_{s r} \mid \gamma_{s r}>\gamma_{t}\right\}<$ $\frac{1}{2 \bar{\gamma}_{s r}} \operatorname{erfc}\left(\sqrt{\gamma_{t}}\right)$. In the case of $P_{b}(\gamma)=b \operatorname{erfc}(a \gamma)$, this bound can easily be generalized to

$$
\mathbb{P}\left\{\mathcal{E}_{s r} \mid \gamma_{s r}>\gamma_{t}\right\}<\frac{1}{\bar{\gamma}_{s r}} \operatorname{berfc}\left(\sqrt{a \gamma_{t}}\right) .
$$

Using the well-known bound $\operatorname{erfc}(z)<e^{-z^{2}}$, we obtain

$$
\mathbb{P}\left\{\mathcal{E}_{s r} \mid \gamma_{s r}>\gamma_{t}\right\}<\frac{b}{\bar{\gamma}_{s r}} e^{-a \gamma_{t}} .
$$

By substituting $\gamma_{t}=\log \left(c_{1} \mathrm{SNR}^{M / a}\right)$, we conclude that

$$
\mathbb{P}\left\{\mathcal{E}_{s r} \mid \gamma_{s r}>\gamma_{t}\right\}<\frac{b}{\bar{\gamma}_{s r}} \frac{1}{c_{1}^{a}} \frac{1}{\mathrm{SNR}^{M}}=\frac{b}{c_{1}^{a} \lambda_{s r}} \frac{1}{\mathrm{SNR}^{M+1}}(16)
$$

Thus $\mathbb{P}\left\{\mathcal{E}_{s r} \mid \gamma_{s r}>\gamma_{t}\right\}=O\left(1 / \mathrm{SNR}^{M+1}\right)$.

Part 3: As seen in (8), $\mathbb{P}\left\{\mathcal{A}_{r} \mid N_{r}=i\right\}$ depends on $\bar{\gamma}_{r d}$ and $\bar{\gamma}_{s d}$ only through their ratio. Hence, this quantity is independent of SNR and $\mathbb{P}\left\{\mathcal{A}_{r} \mid N_{r}=i\right\}=O(1)$.

Combining Parts 1, 2 and 3, we obtain

$$
\begin{aligned}
\mathbb{P}\left(\mathcal{E}_{e 2 e} \mid N_{r}=i\right) & =\underbrace{\mathbb{P}\left\{\mathcal{E}_{s d}, \mathcal{A}_{s} \mid N_{r}=i\right\}}_{O\left(1 / \mathrm{SNR}^{i+1}\right)}+i \times(\underbrace{\mathbb{P}\left\{\mathcal{E}_{r d}, \mathcal{A}_{r} \mid N_{r}=i\right\}}_{O\left(1 / \mathrm{SNR}^{i+1}\right)} \\
& +\underbrace{\mathbb{P}\left\{\mathcal{E}_{r d}, \mathcal{A}_{r} \mid N_{r}=i\right\}}_{O\left(1 / \mathrm{SNR}^{i+1}\right)} \underbrace{\left(1-2 \mathbb{P}\left\{\mathcal{E}_{s r} \mid \gamma_{s r}>\gamma_{t}\right\}\right)}_{O(1)} \\
& +\underbrace{\mathbb{P}\left\{\mathcal{A}_{r} \mid N_{r}=i\right\}}_{O(1)} \underbrace{\mathbb{P}\left\{\mathcal{E}_{s r} \mid \gamma_{s r}>\gamma_{t}\right\}}_{O\left(1 / \mathrm{SNR}^{M+1}\right)}) .
\end{aligned}
$$

Hence, $\mathbb{P}\left\{\mathcal{E}_{e 2 e} \mid N_{r}=i\right\}=O\left(1 / \mathrm{SNR}^{i+1}\right)$.

\section{REFERENCES}

[1] N. Laneman, D. Tse, and G. Wornell, "Cooperative diversity in wireless networks: Efficient protocols and outage behavior," IEEE Trans. Inf. Theory, vol. 50, pp. 3062-3080, Dec. 2004.

[2] J. N. Laneman and G. W. Wornell, "Distributed space-time-coded protocols for exploiting cooperative diversity in wireless networks," IEEE Trans. Inf. Theory, vol. 49, pp. 2415-2425, Oct. 2003.

[3] A. Bletsas, A. Khisti, D. P. Reed, and A. Lippman, "A simple cooperative diversity method based on network path selection," IEEE Journal on Selected Areas in Communications, vol. 24, pp. 659 - 672, Mar. 2006.

[4] T. Wang, R. Wang, and G. Giannakis, "Smart regenerative relays for link-adaptive cooperative communications," in Proc. 40th Annual Conference on Information Sciences and Systems (CISS), Princeton, NJ, 2006.

[5] T. Wang, G. B. Giannakis, and R. Wang, "Smart regenerative relays for link-adaptive cooperation." IEEE Trans. Commun., to appear.

[6] D. Chen and J. N. Laneman, "Modulation and demodulation for cooperative diversity in wireless systems," IEEE Trans. Wireless Commun., vol. 5, pp. 1785-1794, July 2006.

[7] T. Wang, A. Cano, G. B. Giannakis, and J. N. Laneman, "Highperformance cooperative demodulation with decode-and-forward relays," IEEE Trans. Commun., vol. 55, pp. 1427-1438, July 2007.

[8] Z. Yi and I.-M. Kim, "Decode-and-forward cooperative networks with relay selection," in Proc. IEEE Vehicular Technology Conference, VTC Fall, Baltimore, MD, 2007.

[9] P. Herhold, E. Zimmermann, and G. Fettweis, "A simple cooperative extension to wireless relaying," in Proc. International Zurich Seminar on Communications, Zurich, Switzerland, 2004.

[10] F. A. Onat, A. Adinoyi, Y. Fan, H. Yanikomeroglu, J. Thompson, and I. Marsland, "Threshold selection for SNR-based selective digital relaying in cooperative wireless networks." IEEE Trans. Wireless Commun., to appear.

[11] F. A. Onat, Y. Fan, H. Yanikomeroglu, and J. Thompson, "Asymptotic BER analysis of threshold digital relaying in cooperative wireless networks." IEEE Trans. Wireless Commun., to appear.

[12] E. Beres and R. Adve, "Selection cooperation in multi-source cooperative networks," IEEE Trans. Wireless Commun., vol. 7, pp. 118-127, Jan. 2008

[13] A. Goldsmith, Wireless Communications. Cambridge University Press, Cambridge, UK, 2005.

[14] L. Zheng and D. Tse, "Diversity and multiplexing: A fundamental tradeoff in multiple antenna channels," IEEE Trans. Inf. Theory, vol. 49, pp. 1073-1096, May 2003. 